\title{
Tactile Discrimination of Shape: Responses of Rapidly Adapting Mechanoreceptive Afferents to a Step Stroked Across the Monkey Fingerpad
}

\author{
Robert H. LaMotte and Mandayam A. Srinivasan \\ Department of Anesthesiology, Yale University School of Medicine, New Haven, Connecticut 06510
}

Responses of rapidly adapting Meissner corpuscle mechanoreceptive afferent fibers (RAs) to steps of varying shape stroked across the distal fingerpad were recorded from anesthetized monkeys. A series of flat plates were used, each having an increase in thickness (a step) in the middle so that one-half of the plate was thicker than the other. The cross-sectional shape of the step approximated that of a half-cycle sinusoid, $0.5 \mathrm{~mm}$ high. The width (half-cycle wavelength) of the sinusoidal step was varied from 0 to $3.13 \mathrm{~mm}$, producing a series of step shapes that differed in steepness and curvature. These steps could be broadly categorized into 2 groups, "steep" and "gradual." Each step was stroked back and forth under constant compressional force, using a servocontrolled mechanical stimulator.

The RA's response to a step provided a spatial pattern of action potentials in which the occurrence of each impulse corresponded to a position of the step on the skin. This response consisted of a single "burst" of impulses to the sinusoidal portion of the step. Changes in stroke direction, step shape, or velocity of stroking primarily affected the RA discharge rate during the burst, and, less consistently, the spatial width of the burst. For a given step shape and stroke velocity, the discharge rate was greater for strokes from the low to the high side of the step than for strokes from the high to the low side. Discharge rate was greater for steep than for gradual steps and, for a given step, it increased with stroke velocity. All the major features of the responses were interpreted as being due predominantly to the sensitivity of the RA to vertical velocity at the most sensitive spot on its receptive field, together with a sensitivity to the rate of change in skin curvature at that spot. RA discharge rate distinguished not only the gross differences between steep and gradual steps, but also some of the finer differences in sharpness among steep steps. From a comparison with the human capacity for tactile discrimination of the steps, it was concluded that RAs, through their discharge rates, provide primarily "intensive" information about the sharpness of shapes.

\footnotetext{
Received June 16, 1986; revised Nov. 19, 1986; accepted Jan. 8, 1987.

We thank James Whitehouse for assistance throughout the study, David Reddy for computer programs, and Linda Shiffrin for typing the manuscript. This research was supported by PHS Grant 15888 .

Correspondence should be addressed to Robert H. LaMotte, Ph.D., Department of Anesthesiology, Yale University School of Medicine, 333 Cedar Street, New Haven, CT 06510.

Copyright (C) 1987 Society for Neuroscience $0270-6474 / 87 / 061672-10 \$ 02.00 / 0$
}

In the previous paper we described how the shape of a step was represented in the responses of slowly adapting (SA) mechanoreceptive afferent fibers in the monkey when the step was stroked across the distal fingerpad (LaMotte and Srinivasan, 1987). Both intensive (discharge rate) and certain spatial features of the SA's responses to these steps conveyed information about the direction of stroking, the shape of the steps, and the stroke velocity. These results were interpreted as demonstrating that SAs are highly scnsitive to the amount and ratc of change in skin curvature. In the present study, the responses of glabrous skin rapidly adapting (Meissner corpuscle) afferent fibers (RAs) to the same step stimuli are described.

When a step is stroked across the skin, intensive and spatial information about the step is conveyed to the individual RA or SA, primarily through variations in the vertical deflection of the skin. In addition, spatial information about step shape would be available to a spatially distributed population of RAs and SAs in the region of skin deflection under the step. The results of previous studies, however, indicate that RAs and SAs differ in their responses to vertical indentations of the skin and in their capacities to reflect differences in load distribution or skin deflection profiles. First, RAs respond only to changes in vertical skin indentation and not to steady indentation, whereas SAs respond to both (Iggo, 1963; Talbot et al., 1968). The threshold, or minimal velocity of indentation required to evoke a response, varies considerably for RAs, but is typically higher in RAs than in SAs (Knibestöl, 1973; Pubols and Pubols, 1976, 1983). Second, some RAs are more responsive to the edge of a contact surface than to the flat portion of the surface (Phillips and Johnson, 1981; Johansson et al., 1982), but this edge sensitivity is considerably poorer than that of the SAs.

We have interpreted the superior edge sensitivity of SAs as indicating a sensitivity to changes in skin curvature (LaMotte and Srinivasan, 1987). Thus, it seems likely that RAs, with their poorer edge sensitivity, would not convey as much information as do SAs about the curvatures of a shape stroked across the skin. The results of the present study demonstrate that RAs provide less information than SAs about the spatial features of a shape, such as its curvature profile. However, the responses of RAs are better than those of the SAs in discriminating fine differences in sharpness.

\section{Materials and Methods}

Since the procedures are identical to those already described (LaMotte and Srinivasan, 1987), they will be briefly summarized.

Shapes of the steps. Each step was made of transparent epoxy and consisted of a transition in height between 2 planes. In cross section, the shape of the transition was either a right angle (a $90^{\circ}$ step) or re- 
sembled a half-cycle sinusoid that extended from the low side of the step to the high side at the top of the step. The half-cycle wavelength was varied, resulting in changes in step width and curvature, while the step height was constant at $0.5 \mathrm{~mm}$ (see Fig. 1, LaMotte and Srinivasan, 1987). The widths used for steps that were described by human observers as "steep" were $0,0.45,1.195$, and $1.23 \mathrm{~mm}$, and, for steps that were described as "gradual," 3.038 and $3.134 \mathrm{~mm}$.

Applying the steps to the passive fingerpad. A servocontrolled hydraulic mechanical stimulator (LaMotte et al., 1983) was used to apply the step to the fingerpad and to stroke it back and forth at a controlled compressional force of $20 \mathrm{gm} \mathrm{wt}$. The hand was restrained in plasticine, with the finger to be stimulatcd-usually the middle finger-held at a slightly higher position. The monkey finger was restrained by means of a plastic screw glued to the fingernail and sunk into the plasticine. This restrained the fingernail and the rest of the finger, but not the fingerpad. On each trial, one of the steps was selected and brought down onto the skin on the high side of the step, at a spot $9 \mathrm{~mm}$ from the center of the sinusoidal portion. After an interval of $1 \mathrm{sec}$, the step was stroked with constant horizontal velocity from the high side of the step to a locus on the low side, $9 \mathrm{~mm}$ from the center of the sinusoidal portion. After another $1 \mathrm{sec}$ interval, the step was stroked with constant horizontal velocity back to the high side of the step; this was followed by another 1 sec interval, after which another pair of back-and-forth strokes was delivered. At the end of the last stroke, the step was lifted off the skin and returned to its home position, thus ending the trial. The time between successive contacts with the finger (i.c., between trials) was typically $6 \mathrm{sec}$. In some experiments, the stroke velocity was varied, and 2 trials were given at each of the following velocities: $1,1.5,5,10,20$, and $40 \mathrm{~mm} / \mathrm{sec}$. In other experiments, 10 trials were given at a stroke velocity of $10 \mathrm{~mm} / \mathrm{sec}$, with 2 strokes in each direction per trial.

Neurophysiological experiments. Recordings were made from the upper or lower median nerves of 8 Macaca fascicularis monkeys, each weighing 4-6 kg. Action potentials were recorded from single, rapidly adapting mechanoreceptive afferent fibers innervating the fingerpad. All were of the FAI variety, believed to terminate in Meissner corpuscles, and were distinguished from FAII, i.e., Pacinian corpuscle afferent fibers (according to the classification of Vallbo and Johansson, 1984) on the basis of their smaller receptive fields with well-defined boundaries and their insensitivity to remote, low-intensity taps to the table. Although quantitative studics of Pacinian fibcrs wcre not conducted, the responses of 3 Pacinians to hand-held steps stroked across their receptive fields (which included the fingerpad) were not qualitatively different from those of the Meissner corpuscle fibers.

RA receptive fields were centrally located, away from the nail on the volar surface of the distal phalanx. Most receptive fields were located on the third digit, others on the second or fourth digits. Each fiber's receptive field was first mapped with von Frey filaments. When the step stimuli were stroked across the skin, the step's contact area with the skin was videorecorded through a dissection microscope at $20 \times$ magnification so that the position of the step (actually a reference line on the step) with respect to the fiber's von Frey-determined receptive field at each instant of time could be reconstructed from off-line analysis. This also allowed a fiber's responses to different steps to be spatially superimposed. A PDP 11/34 computer recorded the time of occurrence of each action potential, the beginning of each video field, and the beginning and end of each horizonal stroke with the step. In addition, voltage analogs of compressional force and the vertical displacement were sampled every $20 \mathrm{msec}$ and recorded by the computer

Statistical analysis. The main effects of stroke direction, step shape, and stroke velocity on various measures of mean RA responses were tested for significance using repeated-measures analyses of variance. Pairwise comparisons between individual means were made using the Newman-Keuls procedure (Winer, 1971). The significance level was set at 0.05 .

\section{Results}

General features of RA fibers to steps stroked across their receptive fields

Nine RAs were studied under machine-controlled stimulation. Each step was stroked 20 times in each direction along an 18 $\mathrm{mm}$ path. The stroke velocity was $10 \mathrm{~mm} / \mathrm{sec}$ and the contact force was $20 \mathrm{gm}$ wt. The responses of a typical RA, R29, to stroking with step 3 are shown in Figure 1. On each of 20 trials, the step was first stroked from the high to the low side (left column, Fig. 1) and then stroked back again from the low to the high side (right column, Fig. 1). Each tic mark is the instantaneous position on the skin of a reference line on the step at the time of occurrence of each action potential. The reference line was located at the beginning of the convex curvature on the high side of the step (see Fig. 1, LaMotte and Srinivasan, 1987). Thus, the dashed line connecting the von Frey-determined receptive field in Figure $1 A$, with the spatial impulse plot below, indicates that the RA's rate of discharge (Fig. 1C) reached a maximum when the convex part on the high side of the step reached the most sensitive spot within the receptive field. Since the nerve impulses are plotted according to distance (step position) and not time, the stroke from right to left (right half of Fig. 1) starts on the right and ends on the left.

The general features of this RA's response to step 3 were typical of the responses of most of the RAs to each of the steps: (1) There was a transient response to skin stretch at the onset of each stroke in each direction; (2) there was no basal discharge to the flat portion of the step stroked across the receptive field; (3) there was a "burst" of discharge to the sinusoidal portion of the step; (4) there were no consistent differences in the patterns of RA response within the burst that distinguished the direction of stroking. Except for one, all the RAs exhibited a greater discharge rate to step stroking from low to high than to stroking from high to low.

An individual RA's response to repeated strokes with a given step was highly reproducible. Representative responses of 6 RAs to steps 1 and 5 stroked in each direction at $10 \mathrm{~mm} / \mathrm{sec}$ are shown in Figure 2. The responses of 3 RAs are not shown, since they were not tested with all 20 strokes in each direction. The total number of impulses evoked during a vertical indentation to $20 \mathrm{gm}$ wt at $4 \mathrm{~mm} / \mathrm{sec}$ by the flat portion of the step, and during its retraction at $4 \mathrm{~mm} / \mathrm{sec}$, is given for each fiber on the right side of Figure 2. The responses are ranked from top to bottom in order of decreasing mean burst discharge rate evoked by step 1, stroked from low to high. With the exception of R32 and $\mathrm{R} 28$, this order is the same as the ranking according to the relative sensitivity of each fiber to vertical indentation of the skin.

The pattern of discharge within the burst in response to the sinusoidal portion of a given step differed between individual RAs. Also, an individual RA's discharge pattern within the burst typically changed with different stimulus conditions. First, a fiber's pattern of response to the same step was usually slightly different for the 2 stroke directions. Second, the pattern changed with changes in step shape. In general, the responses to a gradual step stroked in either direction were more likely to contain a pause within the burst than were the responses to a steep step. The opposite pattern, consisting of a continuous response within the burst, with a short region of increased discharge, was seen only in the responses to steep steps.

All RAs except R31 exhibited a greater discharge rate to step stroking from low to high (as opposed to from high to low) for a given stroke velocity, and most were more responsive to vertical indentations than to retraction of the skin, suggesting a relationship between sensitivities to these 2 types of stimuli (Fig. 2). However, a weak response to step indentations did not always indicate that there would be a weak response to stroking. R32 gave a weak response to both vertical indentation and retraction, yet exhibited the highest discharge rate of any RA to step stroking. 
A
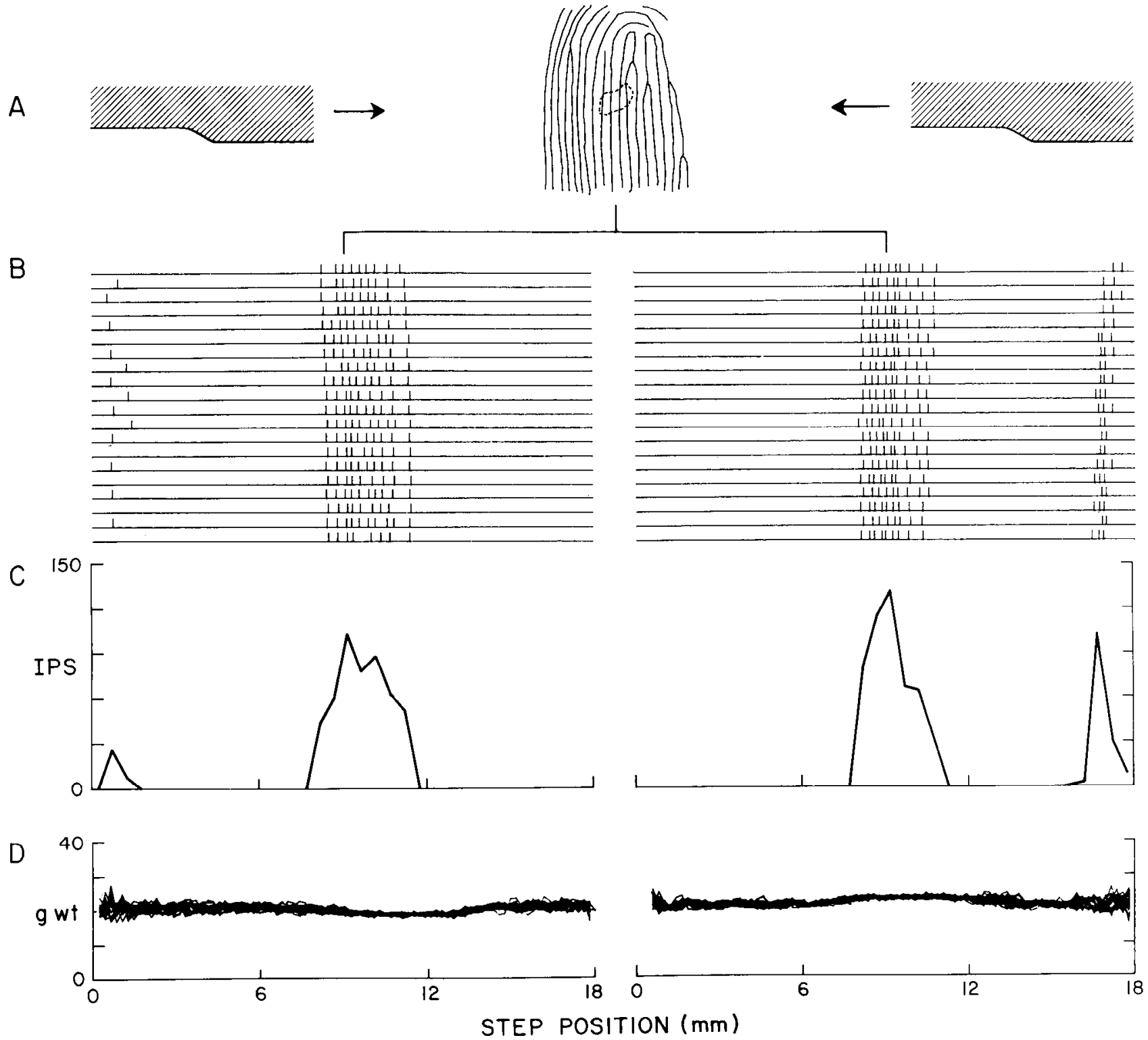

Figure 1. Responses of an RA (R29) to a step stroked back and forth across its receptive field. $A$, Schematic drawing of step 3 shown in crosssectional profile. Only the sinusoidal portion is drawn to the same scale as the rest of the figure; the lateral extent of the flat portions of the step is truncated. In the middle are the papillary grooves on the monkey fingerpad. The circular dotted area is the receptive field of the RA (as determined by a $0.17 \mathrm{gm}$ wt von Frey filament) drawn to the same scale as that for step positions during stroking, indicated below. The branched vertical line indicates the location of the center of the von Frey receptive field on the spatial impulse plots below. $B$, Spatial plots of nerve impulses evoked during stroking of step 3 from the high to low side (left) and from the low to high side (right). Vertical tic marks are the positions of the reference mark on the step at the occurrence of an action potential during a given $18 \mathrm{~mm}$ stroke, each represented by a horizontal line. The reference mark is located at the start of the convex curvature at the top of the step. Note that time begins at the left end for the left panel and at the right end for the right panel. The transient response at the beginning of each stroke was elicited by initial stretch. There were 20 strokes in each direction at 10 $\mathrm{mm} / \mathrm{sec}$. $C$, Iistograms plotting the mean discharge rate (impulses/sec or IPS) per consecutive bins of $0.5 \mathrm{~mm}$ width, averaged from the impulse data above. $D$, Force $(20 \mathrm{gm} \mathrm{wt})$ applied by the step on the finger. Force traces from all the 20 strokes are superimposed.

Responses to hand-held step stimuli. We studied the effect on 5 additional RAs of hand-held steps stroked back and forth along 2 orthogonal axes, one perpendicular and the other parallel to the long axis of the finger. The size of the contact area with the skin was kept about the same along each axis. The patterns of discharge elicited by these hand-held step stimuli stroked at approximately $10 \mathrm{~mm} / \mathrm{sec}$ were qualitatively similar to those elicited during machine-controlled stimulation.

\section{Effect of the shape of a step on $R A$ responses}

For strokes in either direction, the discharge rate of each RA during the burst was greater for the steeper than for the gradual 

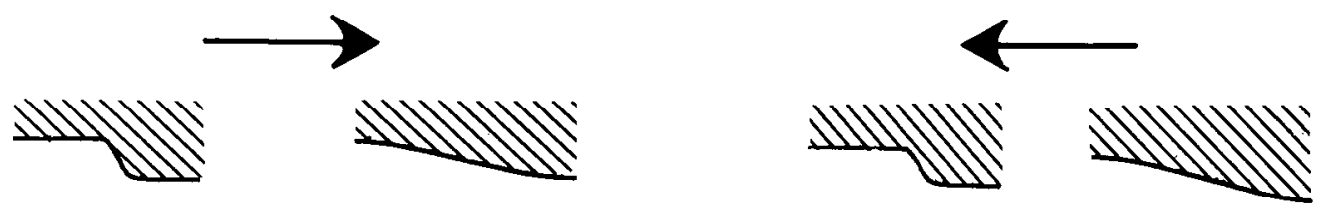

VERTICAL INDENT.
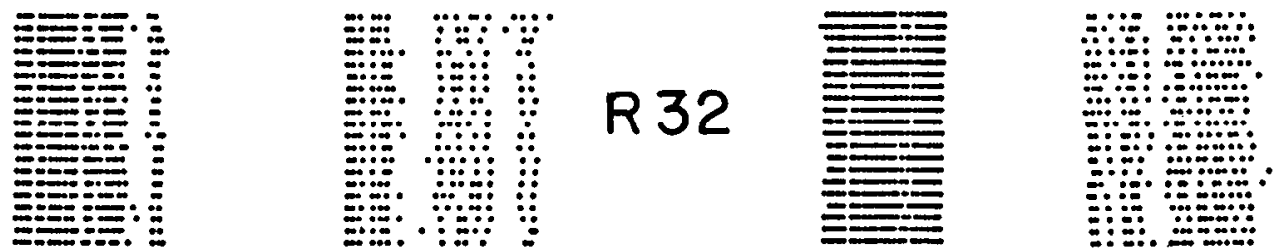

in

out
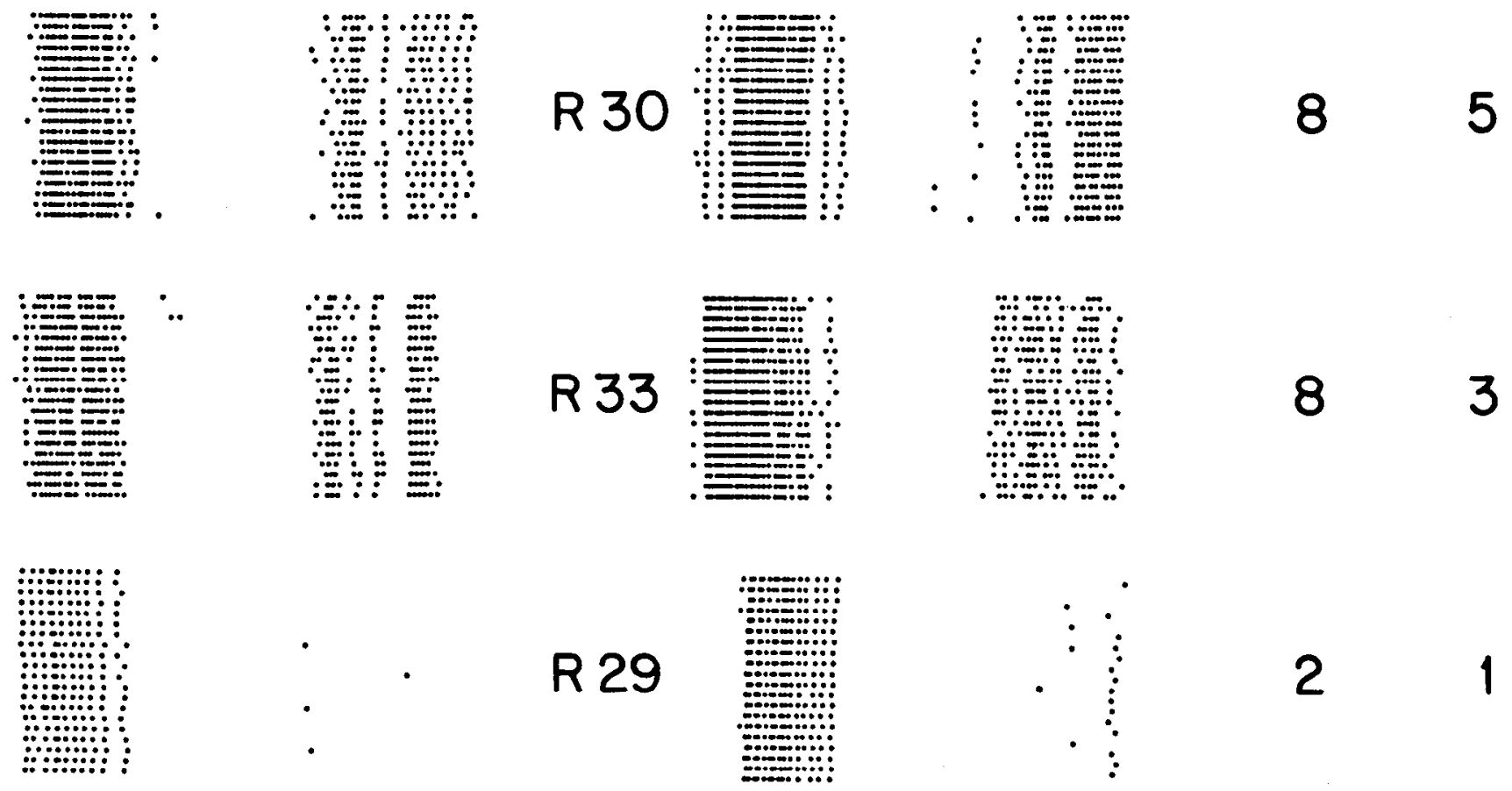

•
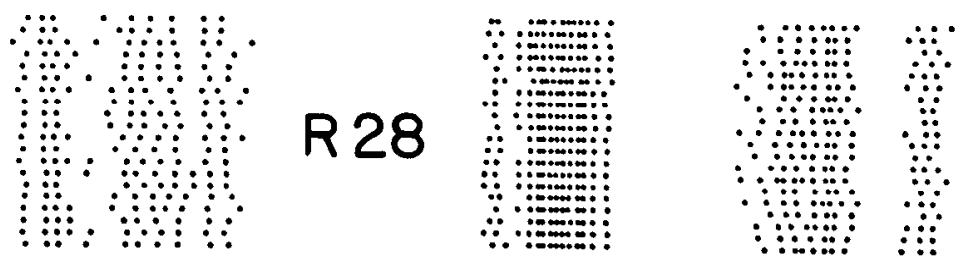

12
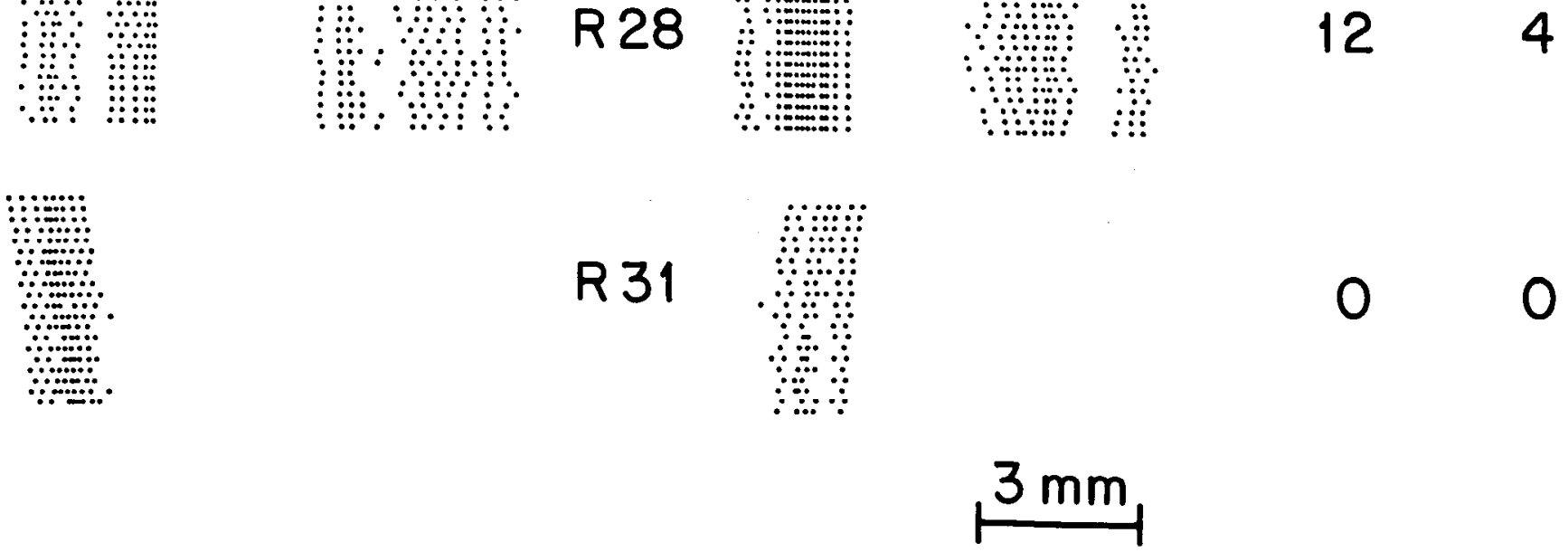

were 20 strokes of $10 \mathrm{~mm} / \mathrm{sec}$ in each direction. Dots indicate positions of the steps (reference marks on the steps) on the skin at the time of occurrence of each action potential. RA responses are ranked from top to bottom in order of decreasing mean discharge rate during the burst for low-to-high strokes under step 1 . On the right side of the figure is the total number of impulses evoked in each RA when a flat plate was vertically indented at $4 \mathrm{~mm} / \mathrm{sec}$ into the skin until a force of $20 \mathrm{gm}$ wt was reached, and then retracted at $4 \mathrm{~mm} / \mathrm{sec}$. 
Figure 3. Effects of changes in step shape on RA responses to steps stroked in each direction. $A-D$, Response measures are shown for an individual RA (R30, dashed line) and the mean of all 8 RAs (solid line) for each step plotted as functions of step width. $A, B$, Discharge rate during the burst (impulses/ $\mathrm{scc})$. $C, D$, Width of the burst $(\mathrm{mm})$. Stroke velocity was $10 \mathrm{~mm} / \mathrm{sec}$.
STROKING : HIGH TO LOW

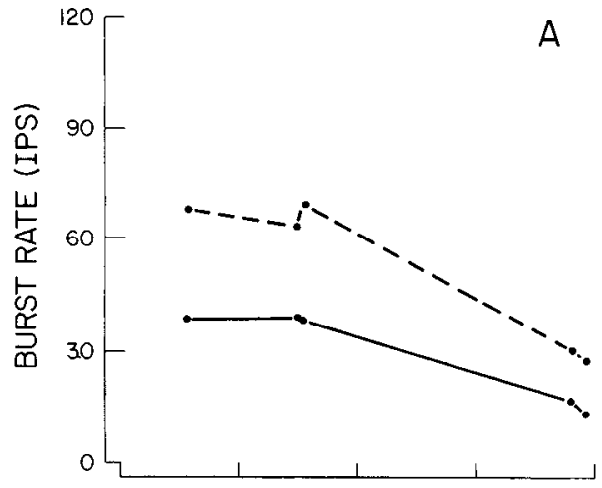

A
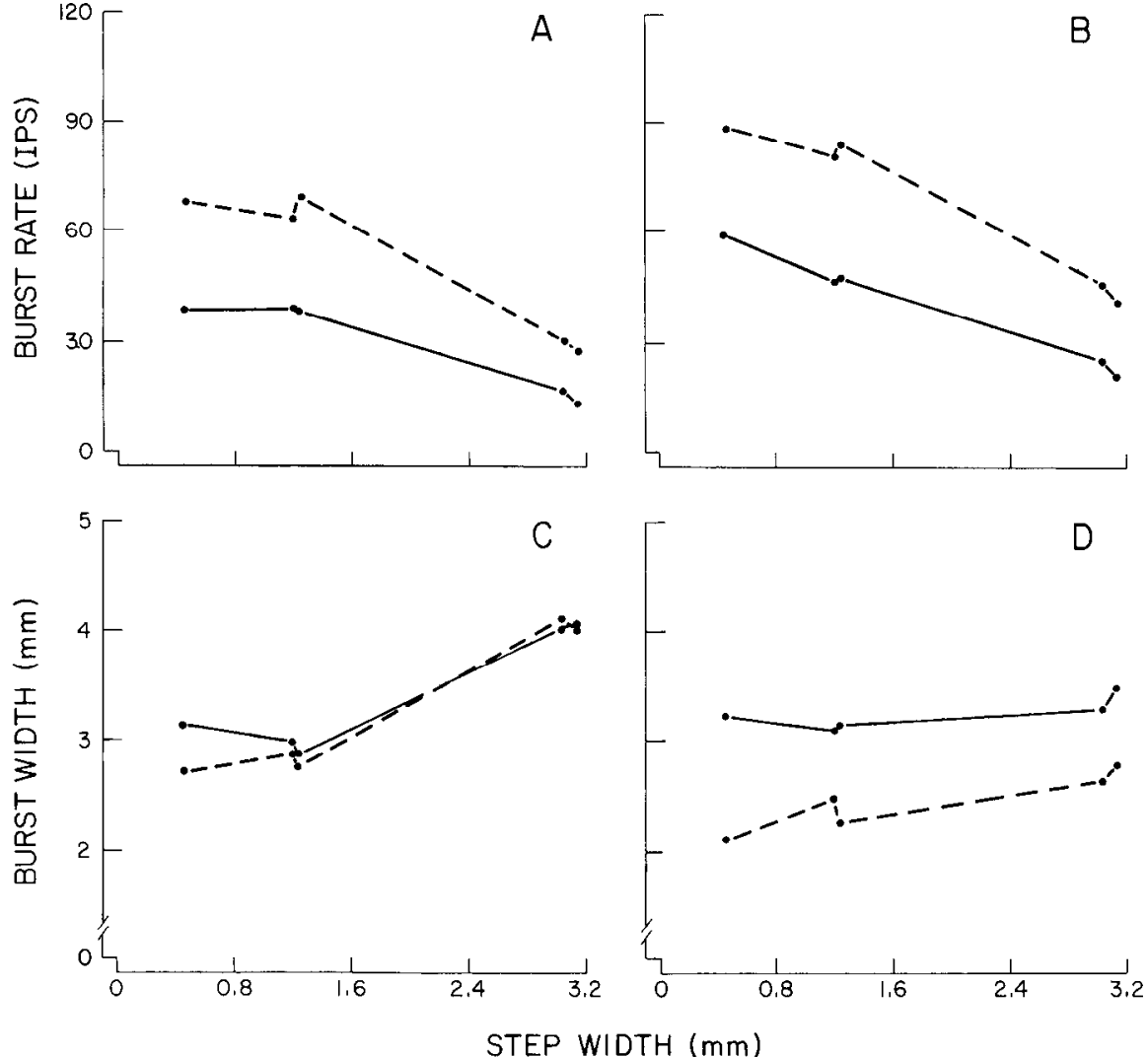

step (Fig. 2). Also, for strokes from high to low, the width of each burst was typically narrower for the steeper than for the gradual step (Fig. 2, left panels), but not for strokes from low to high (Fig. 2, right panels).

Three RAs responded weakly or not at all to the gradual step stroked at $10 \mathrm{~mm} / \mathrm{sec}$. These RAs also responded weakly or not at all to the $4 \mathrm{~mm} / \mathrm{sec}$ vertical indentation (R29 and 31, Fig. 2). One of them, R31, had a higher velocity threshold to vertical indentations than the other RAs. When stroked at $10 \mathrm{~mm} / \mathrm{sec}$, it responded well to step 1 , weakly to step 3 , and not at all to the gradual steps (4 and 5). A stroke velocity of $40 \mathrm{~mm} / \mathrm{sec}$ elicited a response in this fiber to all steps, albeit a weak one to the gradual steps.

In order to obtain average response measures for all RAs, the mean burst width and the mean discharge rate during the burst were first determined for each individual RA on the basis of responses to 20 strokes at $10 \mathrm{~mm} / \mathrm{sec}$ in a given direction to a given step. The mean discharge rate was defined as the number of impulses during the burst divided by the duration of the burst. The means were then averaged for all RAs and plotted as functions of step width in Figure 3 (solid lines). The response measures for an individual RA, R30, are also shown (dashed lines).

The effects of step shape on the mean burst discharge rate and the mean burst width were tested statistically with repeatedmeasures analyses of variance followed by pairwise comparisons between individual means, using the Neuman-Keuls procedure (Winer, 1971). For these and all subsequent tests, the level of significance was set at 0.05 . Data for step 0 were not included in these analyses or plotted in Figure 3, since the step was used only with 4 RAs. Also, 3 RAs did not respond to the gradual steps at $10 \mathrm{~mm} / \mathrm{sec}$ and were not included in the analysis of burst width. For strokes from high to low, step shape had a significant effect on burst discharge rate and on burst width. Steep steps (1-3) had significantly greater rates and narrower bursts than the gradual steps (4 and 5 ). No significant differences in rate or width were found among steps within the categories of steep or gradual. For strokes from low to high, the burst discharge rate for step 1 was significantly greater than those for steps 2 and 3, which, in turn, were significantly greater than those for steps 4 and 5 . There was no significant cffect of step shape on burst width for strokes from low to high.

\section{Effect of stroke velocity on the responses of $R A$ s to different step shapes}

Shown in Figure 4 are the responses of R33 to a steep and a gradual step (steps 1 and 5, respectively) stroked at different velocities. Responses to the first 2 strokes in each direction at velocities of $1,1.5,5,10,20$, and $40 \mathrm{~mm} / \mathrm{sec}$ are shown. The spatial impulse plots were aligned so that the centers of the bursts were in the same horizontal position and only those responses occurring within $5 \mathrm{~mm}$ on either side of a center are displayed (in order to eliminate the effects of skin stretch, which increased in magnitude with decreasing velocity, thereby changing the locus of the burst). R33 responded to the steep step at all velocities, but responded little or not at all to the gradual step at the slower stroke velocities of 1 and $1.5 \mathrm{~mm} / \mathrm{sec}$. For either stroke direction, the steeper the step or the higher the stroke velocity, the higher the burst discharge rate.

The grand means for the discharge rates during the burst (burst rate) and the burst width obtained for 7 RAs are shown in Figure 5. In each panel, the response measures for gradual steps (4 and 5) are represented by dashed lines, and those for steeper steps 


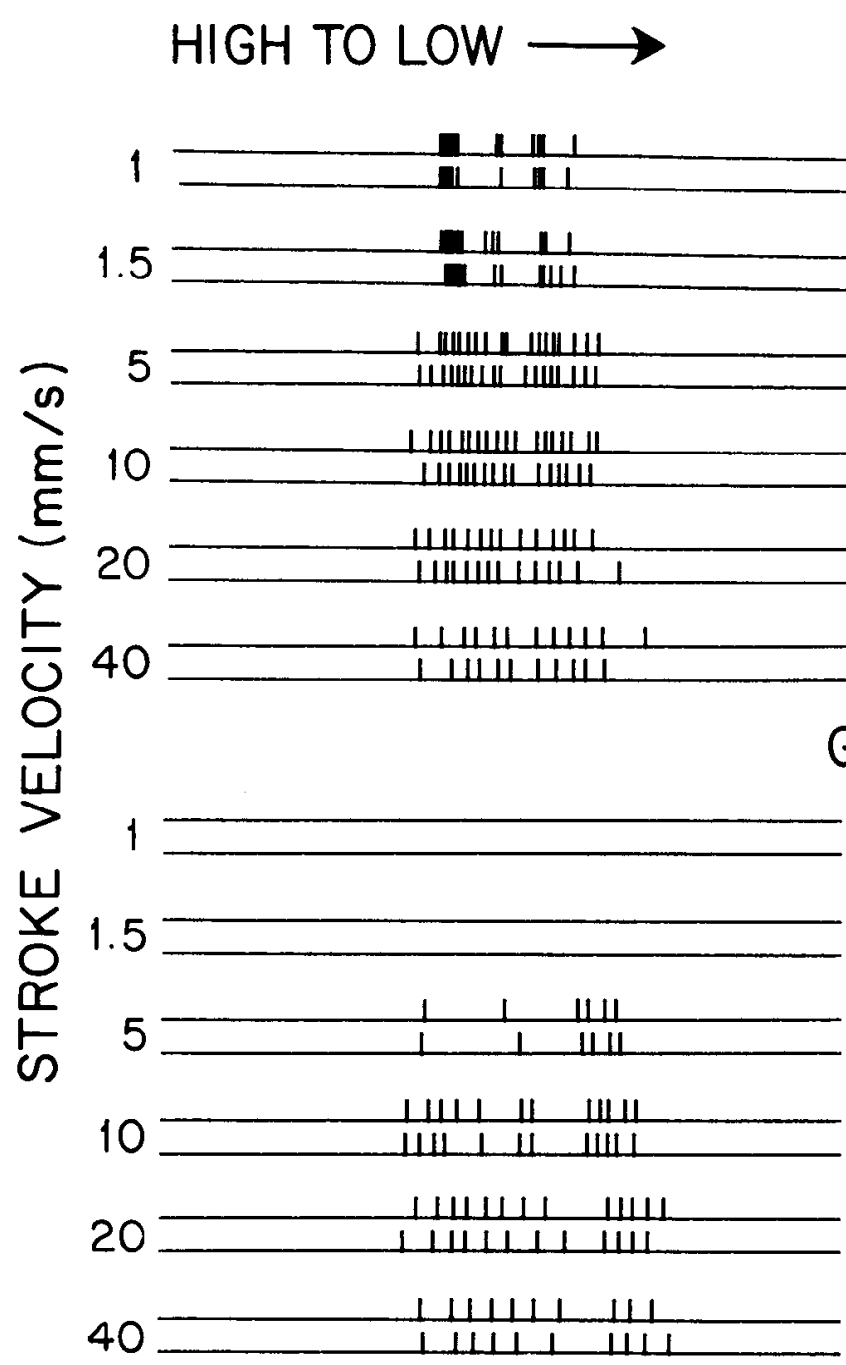

STEEP STEP

(1)

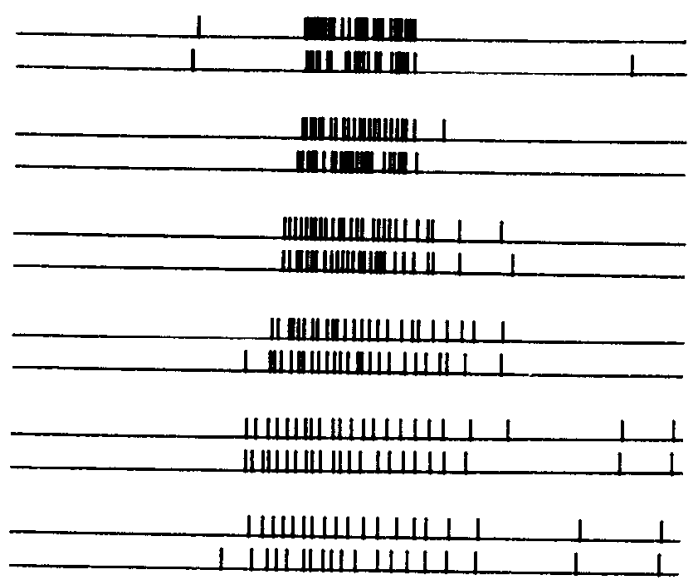

\section{GRADUAL} STEP

(5)

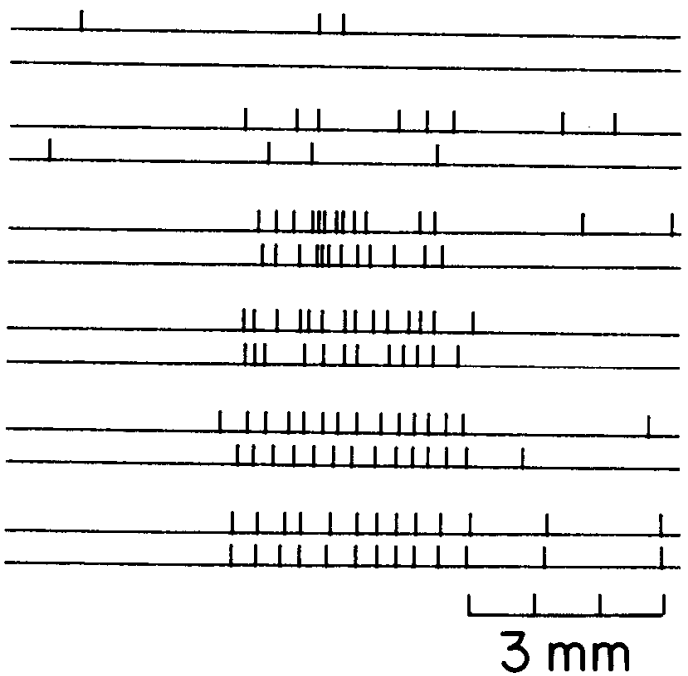

Figure 4. Effects of stroke velocity on an RA's response to a steep and a gradual step. Spatial impulse plots of R33's response to steps 1 and 5 stroked 2 times in each direction at each stroke velocity are shown. The responses to different velocities are arbitrarily aligned with the center of the burst in order to eliminate slight differences in lateral position due to different amounts of skin stretch. Only responses up to $5 \mathrm{~mm}$ on either side of the center of the burst are shown. Note that, even though the impulses were closer together at lower velocities, the discharge rate was actually much lower than that at higher velocities.

(0-3) by solid lines. The analogous curves obtained for each individual $\mathrm{R} \Lambda$ were very similar to those shown here, averaged over 20 strokes for all 7 RAs.

The results of repeated-measures analyses of variance and of Neuman-Keuls comparisons between pairs of individual means for the effects of stroke direction, step shape, and stroke velocity on RA burst discharge rate and burst width are summarized separately below.

Stroke direction. Strokes from low to high produced greater burst discharge rates for a given step and stroke velocity than strokes from high to low. There was no significant effect of stroke direction on burst width.

Step shape. For either stroke direction, step shape had a significant effect on burst discharge rate, but not on burst width. At a given stroke velocity and stroke direction, the burst discharge rate was significantly greater for step 0 than for steps 2 and 3 and greater for steps $0-3$ than for steps 4 or 5 . Rates did not differ between steps 4 and 5 or steps 2 and 3. Only for strokes from low to high was the burst discharge rate significantly greater for step 1 than for steps 2 and 3 .

For either stroke direction, step shape did not have a significant effect on burst width. Nevertheless, for high-to-low strokes, burst widths were generally narrower for steep than for gradual steps at each velocity, except at $40 \mathrm{~mm} / \mathrm{sec}$ (and for step 5 at 1 $\mathrm{mm} / \mathrm{sec}$ ). However, this difference was not statistically significant because 4 of 7 RAs exhibited only 1 or 0 impulses for gradual steps at the slower velocities, and these RAs were not included in the analyses of burst width.

Stroke velocity. Burst discharge rate increased significantly with stroke velocity for both directions. Not shown in Figure 5 is the total number of impulses per burst that, for either direction, also increased with increases in velocity up to $5 \mathrm{~mm} / \mathrm{sec}$ and then decreased slightly with further increases in velocity. Burst width increased significantly with stroke velocity only for 
Figure 5. Effects of stroke velocity on the mean responses of all RAs to different step shapes. Grand means were obtained from mean responses of 8 RAs. Responses to steep steps $(0-3)$ are shown as solid lines, and responses to the 2 gradual steps ( 4 and 5 ) as dashed lines. burst. $C, D$, Mean width of the burst. $A, B$, Mean discharge rate during the
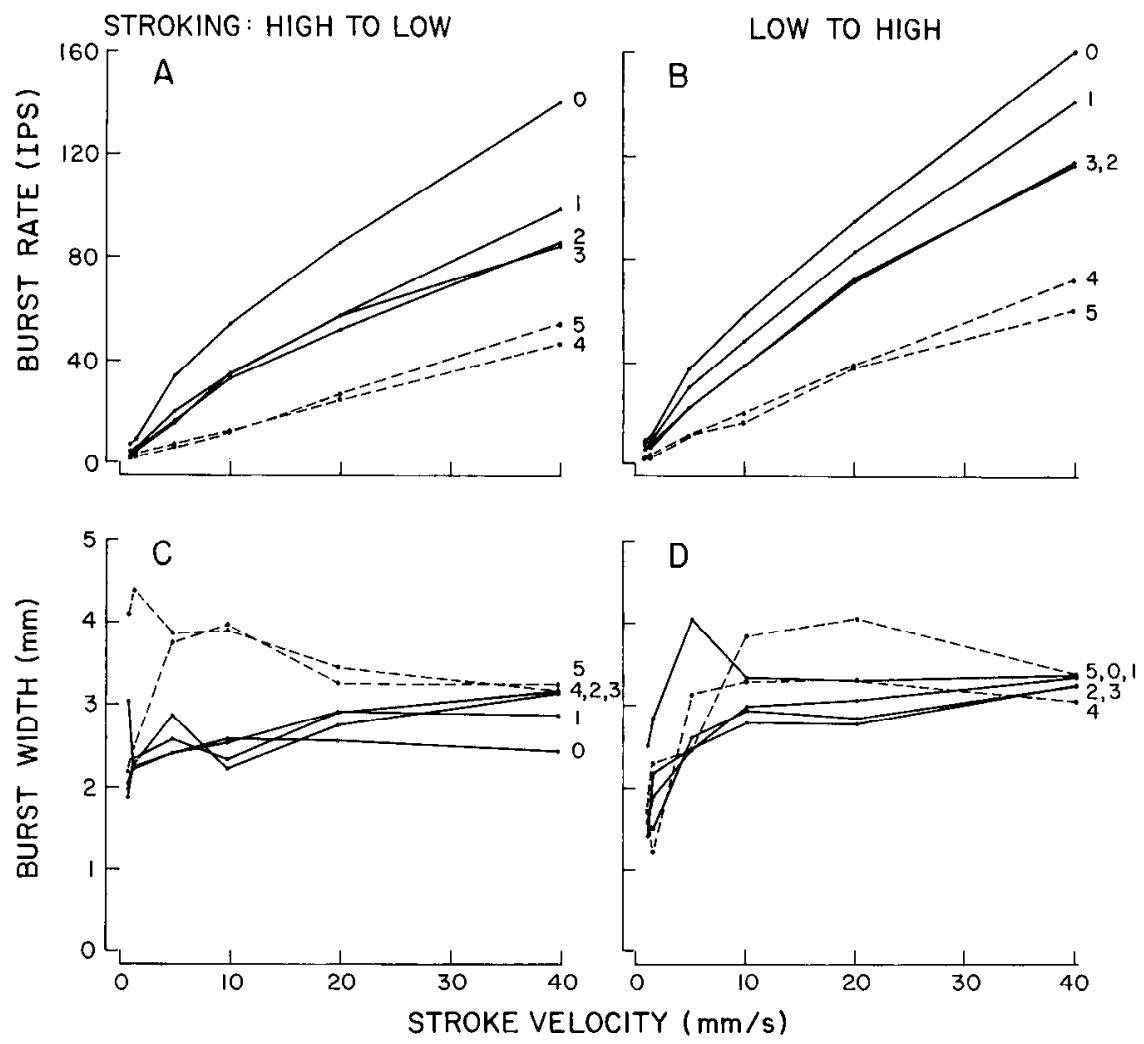

low-to-high strokes, but remained approximately constant for high-to-low strokes.

There was a significant interaction between the effects of step shape and stroke velocity on burst discharge rate. The differences in burst discharge rate were greater between different steps at higher than at lower stroke velocities.

Although for either stroke direction and at a given stroke velocity, the burst discharge rate was greater for a steep than for a gradual step, the discharge rates of the 2 steps would be expected to be equal if the gradual step were stroked at a suf- ficiently greater velocity relative to the stroke velocity of the steep step. For example, the discharge rates would be about the same for steps 3 and 4 (steep and gradual steps, respectively) if step 3 were stroked slowly at $5 \mathrm{~mm} / \mathrm{sec}$ and step 4 more rapidly at about $15 \mathrm{~mm} / \mathrm{sec}$. But within the range of $5-15 \mathrm{~mm} / \mathrm{sec}$, the steep step would invariably evoke a greater discharge rate than the gradual step, even if their velocities differed. Even when the burst discharge rate is the same for 2 steps stroked at different velocities, their patterns of discharge within the burst might differ and serve as a basis for discrimination of step shape.
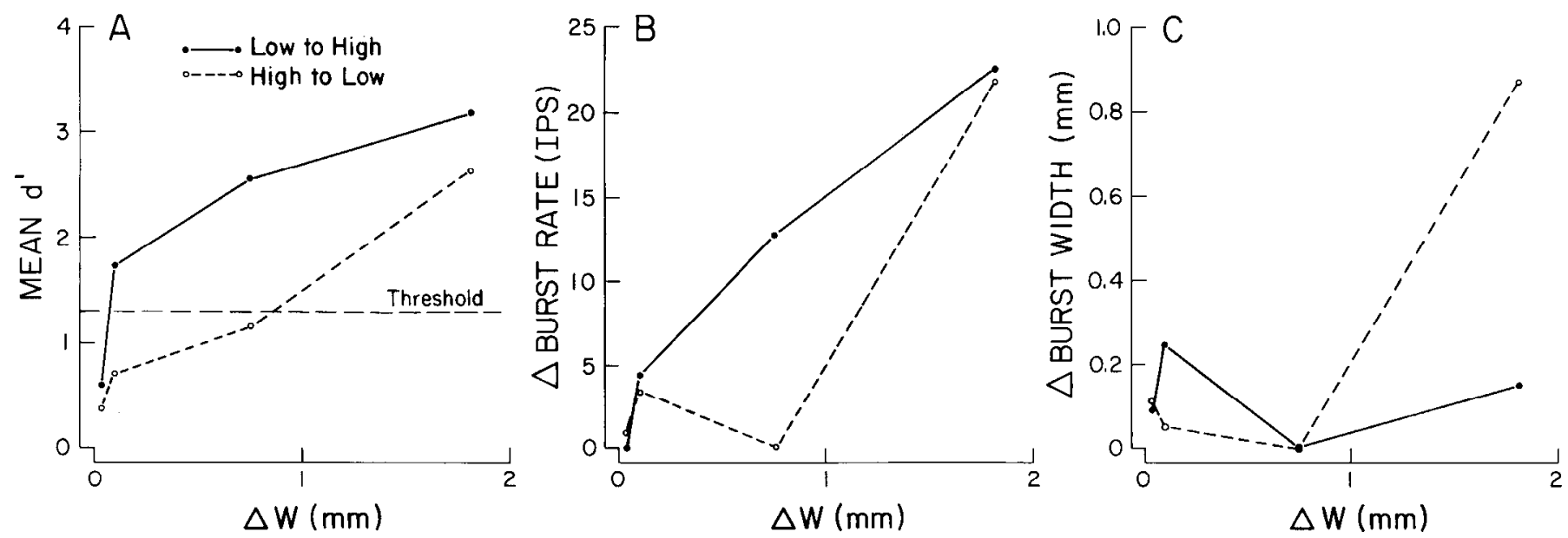

Figure 6. Human sensory discrimination and monkey RA response discrimination of differences in step shapes. Solid and dashed lines represent response measures for step stroking at $10 \mathrm{~mm} / \mathrm{sec}$, respectively, from low to high and from high to low. Differences in step shapes, represented in terms of the differences in step widths $(\Delta W)$, are plotted in order of increasing magnitude, between steps 2 and 3,4 and 5,1 and 2 , and 3 and 4. $A$, Mean $d^{\prime}$ for 5 subjects. $B$, Differences in mean burst discharge rates, averaged from responses of 8 RAs. $C$, Differences in mean burst width obtained from the responses of the same RAs. In 2 instances, slightly negative differences in RA response were arbitrarily plotted as values of 0 . 
However, these patterns differed considerably among RAs even at the same velocity (Fig. 2), and thus are unlikely to provide reliable information.

The following conclusions were drawn from the experiments on the effect of stroke velocity. (1) The burst discharge rate for low-to-high strokes was almost always greater than that for high to low. (2) For a given stroke direction, the steeper the step or the higher the stroke velocity, the greater the burst discharge rate. (3) Burst width remained relatively constant under most stroke velocities.

\section{Comparison of discriminative responses of monkey RAs with sensory discrimination performance in humans}

The capacities of humans to discriminate between different step shapes were determined separately for strokes in each direction and $d^{\prime}$, a measure of sensory discrimination (Johnson, 1980), was computed. The methods and results of these psychophysical tests have been described previously (LaMotte and Srinivasan, 1987). The mean $d^{\prime}$ for 5 subjects is plotted in Figure $6 A$ as a function of the differences in step widths, $\Delta W$, for the step pairs presented in the discrimination task. The $\Delta W$ values (and the associated step pairs) were, from the smallest to the largest, 35 $\mu \mathrm{m}$ (for step 2 versus 3 ), $96 \mu \mathrm{m}$ ( 4 versus 5), $745 \mu \mathrm{m}$ ( 1 versus 2 ), and $1808 \mu \mathrm{m}$ ( 3 versus 4 ). The horizontal dashed line in Figure $6 A$ indicates the $d^{\prime}$ corresponding to the discrimination threshold. Discriminative performance increased with $\Delta W$ and was greatest for discriminations between steps 3 and 4. Also, discrimination was better when stroking from low to high than from high to low.

Analogous statements can be made for differences in the mean rate of discharge during the RA burst response (Fig. $6 \mathrm{~B}$ ). For strokes from low to high, differences in discharge rate, marginal for step 4 versus 5 and increasing with the $\Delta W$ values for steps 1 versus 2 and 3 versus 4 , correlated with the increasing $d^{\prime}$ values for these step pairs. Differences in discharge rate were statistically significant only for steps 1 versus 2 and 3 versus 4 . However, for most RAs (5 out of 7), the burst discharge rate was higher for step 4 than for step 5 . Overall, strokes from low to high evoked significantly greater differences in discharge rate for a given $\Delta W$ than did strokes in the opposite direction. For strokes from high to low, significant differences in discharge rate were present only for the largest $\Delta W$ (step 3 versus 4). This was in accord with human capacities for discrimination.

In contrast with the information about differences in step shape provided by differences in discharge rate, burst width information was less consistent. The mean width of the burst for step 4 was significantly greater than that for step 3 only for strokes from high to low. (The 3 RAs that did not respond to gradual steps at $10 \mathrm{~mm} / \mathrm{sec}$ were not included in the analysis of burst widths.) No significant difference was found for strokes in the opposite direction. These data support the hypothesis that the information RAs convey about step shapes is based primarily on differences in discharge rate.

\section{Discussion}

RA and SA mechanoreceptive afferents innervating primate glabrous skin have been distinguished by their temporal entrainment capacities in response to sinusoidal vibrations (e.g., Talbot et al., 1968) or by their velocity sensitivities and adaptive properties in response to ramps and steady indentations of the skin (e.g., Pubols and Pubols, 1983). In response to sinusoidal vibrations, SAs are most sensitive to low frequencies (less than
$20 \mathrm{~Hz}$ ) while RAs (Meissner corpuscle type) are tuned (at 1 impulse/cycle) at lowest amplitudes at frequencies close to 30 $\mathrm{H} z$ (Talbot et al., 1968). Tuning curves and best frequencies differ for individual RAs.

In response to vertical indentation of the skin, RAs are primarily sensitive only to the velocity of skin indentation and, unlike SAs, which are sensitive to both velocity and displacement, exhibit almost no discharge to static indentation (e.g., Iggo, 1963; Pubols and Pubols, 1983). The discharge rate of an RA increases monotonically with indentation velocity from threshold to saturation (Knibestöl, 1973; Pubols and Pubols, 1976; Franzen et al., 1984). Within this range, the discharge rate of an RA is approximately proportional to the vertical velocity of the skin at its most sensitive spot in the receptive field. That is, for a given ramp indentation of the skin, the number of impulses divided by ramp time is proportional to the displacement (at the end of the ramp) divided by ramp time. Thus, the number of impulses evoked during a skin indentation ramp should be proportional to the vertical displacement of the spot at the end of the ramp, irrespective of the vertical velocity during the ramp. In that case, the profile of skin deflection produced by an object vertically indented into the skin should match the plot of the number of impulses evoked in the RA population as a function of position along the skin. In fact, this was found to be true for a grating pressed into the skin (Phillips and Johnson, 1981). However, owing to the stiffness of the fingerpad, the surface of the skin may not follow the shape of the object, such as a grating with sharp corners and deep recesses. Thus, a faithful representation of the skin deflection profile may give only weak information about the shape of the indenting object. In addition, other factors would act to distort the representation of shape in the RA spatial response profile. First, the discharge rate of the RA is not strictly linearly related to velocity, in that it starts out slowly at threshold and is saturated at higher velocities (e.g., Knibestöl, 1973). Second, since RAs generally respond with few impulses during ramp indentation (as compared with responses of SAs), the presence or absence of even 1 or 2 impulses may produce a significant distortion of the shape of the spatial response profile. These response properties may contribute to the poor spatial resolution of RA responses.

In contrast, the spatial resolution of SAs is considerably greater than that of RAs when Braille patterns are stroked across or gratings vertically indented into the fingerpad (Johnson and Lamb, 1981; Phillips and Johnson, 1981). The responses of SAs and certain RAs to a plate that is indented into the skin is greater when the edge of the plate is applied to the most sensitive spot within the receptive field than if the flat portion of the plate covers the receptive field (Vierck, 1979; Phillips and Johnson, 1981; Johansson et al., 1982). Edge sensitivity of the SAs is excellent, while that of most RAs is poor or nonexistent. We have interpreted the edge sensitivity of SAs and their relatively high degree of spatial resolution as due to their sensitivity to the amount and rate of change in skin curvature (LaMotte and Srinivasan, 1987). By this interpretation, RAs could be said to be less sensitive than SAs to skin curvature change produced by objects indenting the skin (Srinivasan and LaMotte, 1987).

The lesser sensitivity of RAs to curvature under vertical indentations as compared to that of SAs is also reflected in the simpler pattern of RA discharge to a step stroked back and forth across the fingerpad. SAs exhibited a basal discharge to the flat portion of the plate, which was then interrupted by a sequence 


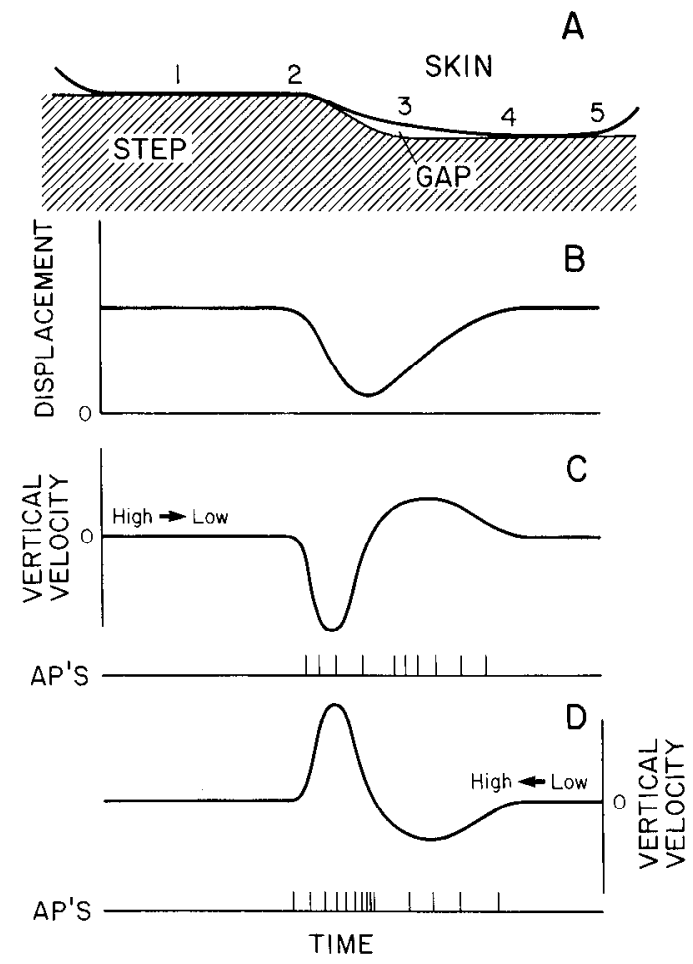

Figure 7. Model of the relationship between the velocity of vertical skin displacement and the pattern of discharge in a hypothetical RA when a finger is actively stroked over a stationary step. $A$, Schematic: of the profile of skin deformation against the step (step 3). Numbers refer to certain successive positions of the most sensitive spot within an RA's receptive field as it is moved from the high to low side of the step (from position 1 to 5 ) or from low to high $(5$ to 1$)$. $B$, Schematic of the changes in vertical displacement of the skin at the most sensitive spot when the RA's receptive field reaches each numbered position on the step. $C$, Schematic of negative (skin retraction) and positive (skin indentation) velocities of skin displacement produced when the finger is moved from the high to the low side of the step (positions 1 to 5) and the resulting action potentials $(A P$ 's $)$ of an RA response. $D$, Schematic of the velocities of skin displacement produced when the finger is moved from the low to high side of the step (positions 5-1) and the corresponding RA response.

of burst-pausc-burst in response to strokes from high to low, and pause-burst-pause to strokes from low to high. RAs exhibited no basal discharge and only a single burst to the sinusoidal portions of the steps, regardless of stroke direction. Thus, SAs and not RAs consistently provided direct information about the variation in curvature across the step profile.

The discharge pattern within the burst was variable among RAs. This was perhaps a reflection of individual differences in their responses to vertical indentation of the skin. Response thresholds for indentation velocity can vary widely, for example, from below $4 \mathrm{~mm} / \mathrm{sec}$ to as high as $39 \mathrm{~mm} / \mathrm{sec}$ (cf. this study; Knibestöl, 1973). The instantaneous rate of discharge during skin indentation is decreasing for most RAs, constant for others, and, in a few, may even be increasing (Pubols and Pubols, 1976, 1983). Finally, RAs differ in their responses to the retraction of a stimulus from the indented skin. In response to retraction, some $\mathrm{R} \Lambda \mathrm{s}$ exhibit only a brief response that is less than their response to indentation, while others exhibit an equal or greater response, and some exhibit no response at all (Pubols, 1980). The diversity of responses to indentations or retractions may help to explain our finding that the pattern of discharge within the burst response to the sinusoidal portion of the step differed from one RA to the next. Variations in step shape and in the dircction as well as the velocity of stroking would be cxpcctcd to produce a variety of vertical indentations and retractions of the skin within the RA's receptive field, and consequently a variety of discharge patterns.

In the following section, the pattern of RA response to a step stroked across the skin is interpreted as being due predominantly to the sensitivity of the RA to alterations in the vertical velocity of skin deflection at the most sensitive spot within the RA's receptive field.

\section{Model of $R A$ responses to step stroking}

For the sake of simplicity, we will assume that RAs are primarily responsive only to the vertical velocity of the most sensitive spot within the receptive field. Consider, then, what happens when the fingerpad is actively stroked across a stationary step that is facing upward, while action potentials are recorded from an $\mathrm{R} \Lambda$ whose receptive field is centrally located within the area of contact between the finger and the step. (The following also applies when the step is stroked across a passive finger.) The approximate profile of deflection of the skin by the plate, as estimated from video recordings in which a light source was placed to the side and behind the step, is shown in Figure $7 \mathrm{~A}$. The profile of skin deflection (Fig. $7 B$ ), defined as the deviation from the normal resting position, is minimal beneath the gap. Vertical displacement of the skin is assumed to be approximately the same in either direction. The velocity of indentation and evoked action potentials in a hypothetical RA are plotted separately for movements of the fingerpad from the high to the low side of the step (Fig. $7 C$ ) and from the low to the high (Fig. $7 D$ ). The RA is assumed to be the kind that responds better to skin indentation (positive velocity) than to skin retraction (negative velocity).

Stroking the finger "off the step" (from the high to low side). As the fingerpad moves between positions 1 and 2 (Fig. 7A), the skin at the RA's most sensitive spot remains at a constant depth of indentation ( 0 vertical velocity), and therefore the RA does not respond. After position 2 is reached, the skin begins to retract, resulting in a negative velocity, and an "off" response is evoked (Fig. 7C). The magnitude of the vertical velocity of retraction first increases and then decreases to 0 . At this point, when retraction is maximal, the skin is closer to its resting state, and thus there is no RA discharge. Next, the indentation phase begins again as position 4 is approached and vertical velocity turns positive, evoking a more vigorous response in the RA. When velocity again falls back to 0 and displacement becomes steady (between positions 4 and 5), the fiber becomes silent.

Stroking the finger "onto the step" (from the low to high side). As the finger is moved from position 5 to 1 , there is initially no activity in the RA, as displacement remains constant (Fig. 7D). There is an "off" response between positions 4 and 3 to the negative velocity (retraction). Then a greater indentation velocity than that produced by strokes from high to low results in a greater "on" response, which then ceases when displacement again becomes steady.

The major difference in the stimulation between strokes in the 2 directions is that the vertical skin displacement produced by the step is first rapidly retracting, then slowly indenting, for a stroke from high to low, or first slowly retracting, then rapidly indenting, for a stroke from low to high. Since most RAs respond better to indentation than to retraction, a stroke from low to 
high evokes a greater discharge rate because of the greater indentation velocity for strokes in that direction.

The effects of stroke velocity and step shape on $R A$ responses. The pattern of discharge in RAs would also be expected to reflect variations in vertical indentation and retraction velocity produced by variations in stroke velocity or step shape. Since the skin profile appears to be approximately the same for different stroke velocities, the higher the velocity, the higher the vertical velocity during indentation and retraction, causing correspondingly higher discharge rates in the RA. Also, for a given stroke velocity, a gradual step produces a more gradual change in skin displacement and hence a milder variation in vertical velocity than a steep step, which might result in a brief pause between "on" and "off" responses (for those RAs responsive to both indentation and retraction). In response to a steep step, the pause within the burst of impulses is typically absent, suggesting that the "on" and "off" responses blend together. Thus, for either stroke direction, the steeper the step or the greater the stroke velocity, the higher the resulting vertical indentation or retraction velocities, causing a higher burst discharge rate. However, in spite of the skin deflection profile's being almost the same under, say, steps 1 and 2, the burst discharge rates, especially for low-to-high strokes, are significantly different. The presence of a highly significant interaction between the effects of stroke velocity and step shape in both stroke directions can be interpreted as simply being due to the sensitivity of the RA to the rate of change of skin curvature at the most sensitive spot. In addition, the steeper (sharper) steps possibly generate subtle vibratory stimuli to which RAs differentially respond, particularly at higher stroke velocities.

Other factors influencing discharge pattern may reflect characteristics of the receptor, such as a sensitivity to skin stretch, a nonlinear relation between discharge rate and velocity, the existence of multiple sensory spots within the RA's receptive field, or varying degrees of sensitivity to the amount or rate of change in skin curvature, as well as to subtle vibrations produced by the sharper steps. For example, there is a response to initial stretch during the beginning of a stroke, even though the vertical velocity remains at 0 . Thus, the model set forth in Figure 7 cannot alone account for all the variations in pattern of discharge from one RA to the next, or from one stimulus condition to the next. However, the model can explain all the major features in a simple manner. This model can easily be used for quantitative predictions of responses under arbitrary stimuli if the velocity threshold and the sensitivity of the RA are known, as well as the skin deflection profile under the stimulus.

\section{Candidate neural codes for step shape}

RA response, plotted as a function of step position during stroking, contains spatial information in the width of the burst and intensive information in the discharge rate during the burst. If the spatial response profile (Fig. $1 C$ ) is interpreted as representative of how a spatially distributed population of RAs responds to step stroking, then the burst width indicates the area of skin containing active RAs. When stroking from the high to low side of the step, the burst width was greater for gradual than for steep steps at most stroke velocities, but there was no consistent difference in burst width for strokes in the opposite direction. In contrast, the spatial response profile of SA responses contained significant spatial information about the difference between steep and gradual steps for strokes in both directions and at most velocities (LaMotte and Srinivasan, 1987). However, the intensive information about fine differences in step shapes contained in the RA discharge rate correlated better than that of SA with the capacities of humans to discriminate these differences in the sharpness of the steps. Among all the intensive and spatial features of mean SA and RA responses, the RA burst discharge rate was the only feature that could explain the human discrimination of step 1 from 2 for low-to-high strokes. Also, 5 out of 7 RAs had a higher burst discharge rate for step 4 than for 5, indicating that the human discrimination of those steps might be based on the RA burst discharge rate. Thus, under stroking, RAs provide better intensive information about differences in shapes related to sharpness, while SAs provide better spatial information.

\section{References}

Franzen, O., F. Thompson, B. Whitsel, and M. Young (1984) Peripheral coding mechanisms of touch velocity. In Somatosensory Mechanisms, C. von Euler, O. Franzen, U. Lindblom, and D. Ottoson, eds., pp. 213-226, Macmillan, London.

Iggo, A. (1963) An electrophysiological analysis of afferent fibres in primate skin. Acta Neuroveg. 24: 225-240.

Johansson, R. S., U. Landström, and R. Lundström (1982) Sensitivity to edges of mechanoreceptive afferent units innervating the glabrous skin of the human hand. Brain Res. 244: 27-32.

Johnson, K. O. (1980) Sensory discrimination: Decision process. J. Neurophysiol. 43: 1771-1792.

Johnson, K. O., and G. D. Lamb (1981) Neural mechanisms of spatial tactile discrimination: Neural patterns evoked by Braille-like dot patterns in the monkey. J. Physiol. (Lond.) 310: 117-144.

Knibestöl, M. (1973) Stimulus-response functions of rapidly adapting mechanoreceptors in the human glabrous skin area. J. Physiol. (Lond.) 232: 427-452.

LaMotte, R. H., and M. A. Srinivasan (1987) Tactile discrimination of shape: Responses of slowly adapting mechanoreceptive afferents to a step stroked across the monkey fingerpad. J. Neurosci. 7: 16551671.

I aMotte, R. H., G. M. Whitehouse, C. J. Robinson, and F. Davis (1983) A tactile stimulator for controlled movements of textured surfaces across the skin. J. Electrophysiol. Techniques 10:1-17.

Phillips, J. R., and K. O. Johnson (1981) Tactile spatial rcsolution. II. Neural representation of bars, edges, and gratings in monkey primary afferents. J. Neurophysiol. 46: 1192-1203.

Pubuls, B. H., Jr. (1980) On- versus off-responses of racoon glabrous skin rapidly adapting cutaneous mechanoreceptors. J. Neurophysiol. 43: $1558-1570$.

Pubols, B. H., Jr., and L. M. Pubols (1976) Coding of mechanical stimulus velocity and indentation depth by squirrel monkey and raccoon glabrous skin mechanoreceptors. J. Neurophysiol. 39: 773-787.

Pubols, B. H., Jr., and L. M. Pubols (1983) Tactile receptor discharge and mechanical properties of glabrous skin. Fed. Proc. 42: 25282535 .

Srinivasan, M. A., and R. H. LaMotte (1987) Tactile discrimination of shape: Responses of slowly and rapidly adapting mechanoreceptive afferents to a step indented into the monkey fingerpad. J. Neurosci. 7: 1682-1697.

Talbot, W. H., I. Darian-Smith, H. H. Kornhuber, and V. B. Mountcastle (1968) The sense of flutter-vibration: Comparison of the human capacity with response patterns of mechanoreceptive afferents from the monkey hand. J. Neurophysiol. 31: 301-334.

Vallbo, A. B., and R. S. Johansson (1984) Properties of cutaneous mechanoreceptors in the human hand related to touch sensation. Hum. Neurobiol. 3: 3-14.

Vierck, C. J. (1979) Comparison of punctate, edge and surface stimulation of peripheral, slowly adapting cutaneous afferent units of cat. Brain Res. 175: 155-159.

Winer, B. J. (1971) Statistical Principles in Experimental Design, McGraw-Hill, New York. 